Int. J. Dev. Biol. 62: 311-318 (2018)

https://doi.org/10.1387/ijdb.180092ct

\title{
Real time dynamics of $\beta$-catenin expression during Hydra development, regeneration and Wnt signalling activation
}

\author{
ROBERTA IACHETTA ${ }^{1}$, ALFREDO AMBROSONE ${ }^{1, \#, ~ A L E X A N D E R ~ K L I M O V I C H ~}{ }^{2}$, JÖRG WITTLIEB ${ }^{2}$, \\ GIADA ONORATO ${ }^{1}$, ALESSIA CANDEO ${ }^{3}$, COSIMO D'ANDREA ${ }^{3,5}$, DANIELA INTARTAGLIA ${ }^{1, \# \#, ~ N U N Z I A ~ S C O T T I ~}{ }^{6}$, \\ MARTINA BLASIO', ANGELA TINO' ${ }^{1}$ ANDREA BASSI ${ }^{3,4}$ and CLAUDIA TORTIGLIONE*,1 \\ ${ }^{1}$ Istituto di Scienze Applicate e Sistemi Intelligenti "E.Caianiello", Consiglio Nazionale delle Ricerche, Pozzuoli, Italy, \\ ${ }^{2} Z$ Zoological Institute, Christian-Albrechts-University of Kiel, Kiel, Germany, ${ }^{3}$ Dipartimento di Fisica, Politecnico di \\ Milano, Milano, Italy, ${ }^{4}$ Istituto di Fotonica e Nanotecnologie, Consiglio Nazionale delle Ricerche, Milano, Italy, ${ }^{5}$ Center \\ for Nano Science and Technology, Italian Institute of Technology, Milano, Italy and ${ }^{6}$ Istituto di Bioscienze e Biorisorse, \\ Consiglio Nazionale delle ricerche, Portici, Italy
}

\begin{abstract}
Understanding the dynamic cellular behaviours driving morphogenesis and regeneration is a long-standing challenge in biology. Live imaging, together with genetically encoded reporters, may provide the necessary tool to address this issue, permitting the in vivo monitoring of the spatial and temporal expression dynamics of a gene of interest during a variety of developmental processes. Canonical Wnt/ $\beta$-catenin signalling controls a plethora of cellular activities during development, regeneration and adulthood throughout the animal kingdom. Several reporters have been produced in animal models to reveal sites of active Wnt signalling. In order to monitor in vivo Wnt/ $\beta$-catenin signalling activity in the freshwater polyp Hydra vulgaris, we generated a $\beta$-cateGFP transgenic $\boldsymbol{H y d r a}$, in which eGFP is driven by the $H y d r a$-catenin promoter. We characterized the expression dynamics during budding, regeneration and chemical activation of the Wnt/ $\beta$-cat signalling pathway using light sheet fluorescence microscopy. Live imaging of the $\beta$-cat-eGFP lines recapitulated the previously reported endogenous expression pattern of $\beta$-catenin and revealed the dynamic appearance of novel sites of Wnt/ $\beta$-catenin signalling, that earlier evaded detection by mean of in situ hybridization. By combining the Wnt activity read-out efficiency of the $\beta$-catenin promoter with advanced imaging, we have created a novel model system to monitor in real time the activity of Hydra $\beta$-cat regulatory sequences in vivo, and open the path to reveal $\beta$-catenin modulation in many other physiological contexts.
\end{abstract}

KEY WORDS: Hydra, live imaging, Wnt, $\beta$-catenin reporter, light sheet fluorescence microscopy

Wnt signalling is a highly conserved signalling pathway that plays crucial role in the animals by controlling the embryonic development, tissue homeostasis and stem cell maintenance in all metazoans (Lim and Nusse, 2013). As expected, disruption or aberrant activation at any level of this complex pathway can lead to developmental abnormalities and diseases, including cancer (Anastas and Moon, 2013, Barker and Clevers, 2006). Since the initial discovery, over the last 30 years, biochemical and genetic analyses performed on vertebrate model organisms and cell cultures dissected the pathway in its single components, and identified large families of
Wnt ligands, receptors, co-receptors and modulators (http://www. stanford.edu/group/nusselab/cgi-bin/Wnt/). Wnt signals are classified as canonical or non-canonical based on the capacity of the former to induce ectopic axis in Xenopus embryos (McMahon and Moon, 1989) and to transform mouse mammary cells (Wong et al., 1994). The canonical pathway is also named the $\mathrm{Wnt} / \beta$ catenin pathway, due to the central role played by the effector $\beta$-catenin

Abbreviations used in this paper: ALP, alsterpaullone; GFP, green fluorescent protein; LSFM, light sheet fluorescence microscopy.

\footnotetext{
*Address correspondence to: Claudia Tortiglione. Via Campi Flegrei 34, 80078 Pozzuoli, Italy. Tel: +39 0818675306 . Fax: +39 0818675126.

E-mail: claudia.tortiglione@cnr.it - web: http://www.tortiglione.com/nanobiomoleculargroup/Sito/Home.html - iD https://orcid.org/ 0000-0003-1447-7611

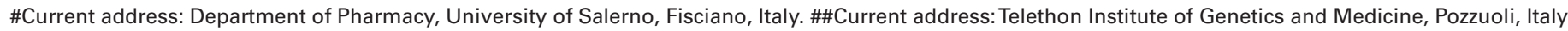

Supplementary Material (fifteen figures) for this paper is available at: https://doi.org/10.1387/ijdb.180092ct

Submitted: 13 March, 2018; Accepted: 23 April, 2018.

ISSN: Online 1696-3547, Print 0214-6282 
( $\beta$-cat) and a destruction complex that controls its stability and activity. Binding of Wnt ligand to receptors of the frizzled family initiates a cascade of events finally resulting in the inhibition of the activity of the destruction complex and $\beta$-cat stabilization. Stabilized $\beta$-cat accumulates in the cytoplasm and then enters the nucleus where it interacts with members of the T-cell factor/lymphoid enhancer factor (TCF/LEF) transcription factor family, ensuring efficient activation of Wnt signalling target genes (Clevers, 2006, MacDonald et al., 2009).

While biochemical and genetic players of canonical Wnt pathway are well characterized during animal development, their temporal and spatial activation in differentiated cells of adult organisms is strictly controlled by mechanisms that are yet not well understood. The fundamental core of the Wnt pathway (Wnt, Frizzled, and downstream effectors) is evolutionarily ancient and is extant in the early-emerging multicellular animals including Cnidaria, in which it mediates axial patterning (Guder et al., 2006, Holstein, 2012, Lengfeld et al., 2009). In the freshwater polyp Hydra vulgaris, the expression of Wnt genes is localized to a small region at the apical tip of the hypostome. This localized expression is critical for setting up and maintaining the primary body axis in intact polyps, and during budding and regeneration processes (Guder et al., 2006, Hobmayer et al., 2000, Lengfeld et al., 2009). In particular, during very early head regeneration, its enhanced expression labels the new developing head structures. Remarkably, other transcriptional components of Wnt/ $\beta$-catenin signalling such as Tcf and $\beta$-cat are transcriptionally upregulated earlier than Wnt during budding and regeneration (Gee et al., 2010, Hobmayer et al., 2000, Petersen et al., 2015) indicating their involvement in establishing a localized signalling centre. The fundamental role played by $\beta$-cat in the gene regulatory network underlying head regeneration has been thoroughly dissected over the last decade (Chera et al., 2009,
Guder et al., 2006, Hobmayer et al., 2000, Lengfeld et al., 2009, Petersen et al., 2015). Within the first 30-60 min after dissection the loss of endodermal cell integrity and the movement of ectodermal cells determinate the wound closure. During this pre-patterning phase, up to $12 \mathrm{~h}$, specific gene networks are activated (Gufler et al., 2018). In particular, nuclear $\beta$-cat up-regulates a set of target genes in a position independent manner. Later on, $\beta$-cat takes action in the transition from pre-patterning to differentiation of terminal head- and foot-specific structures, controlling at translational level specific transcription factors. The mechanisms underlying activation of pathway components in head regenerates during the pre-patterning phase are still unclear, and may possibly rely on Wnt ligands released by amputated cells (Chera et al., 2009), which activate the pathway or may depend on the modulation of casein kinase which in turn upregulates $\beta$-cat and enhances Wnt transcription (Nakamura et al., 2011, Petersen et al., 2015).

While in the hypostome of an adult Hydra $\beta$-cat is located in the cell nuclei (Broun et al., 2005) a predominantly cytoplasmic $\beta$-cat localization is observed in other body regions. It suggests that in contrast to its role as a transcriptional co-factor in the hypostome, $\beta$-cat may be involved in other non transcriptional functions, such as in mediating cell-cell adhesion via adherents junction contacts (Hobmayer et al., 2000, Hobmayer et al., 1996).

The dynamic regulation of the $\beta$-cat gene expression has been tackled by manipulation techniques, such as treatment with alsterpaullone (ALP), a specific inhibitor of GSK-3 $\beta$, (Leost et al., 2000). Treatment of Hydra with ALP caused elevated level of nuclear $\beta$-cat throughout the body column, whose cells acquired head organizer inducing capacity providing evidence of $\beta$-cat role in the head organizer (Broun et al., 2005). These observations, made on fixed samples, provide only "snapshots", from which the entire dynamic processes of regulation can be deduced. This inferior lack
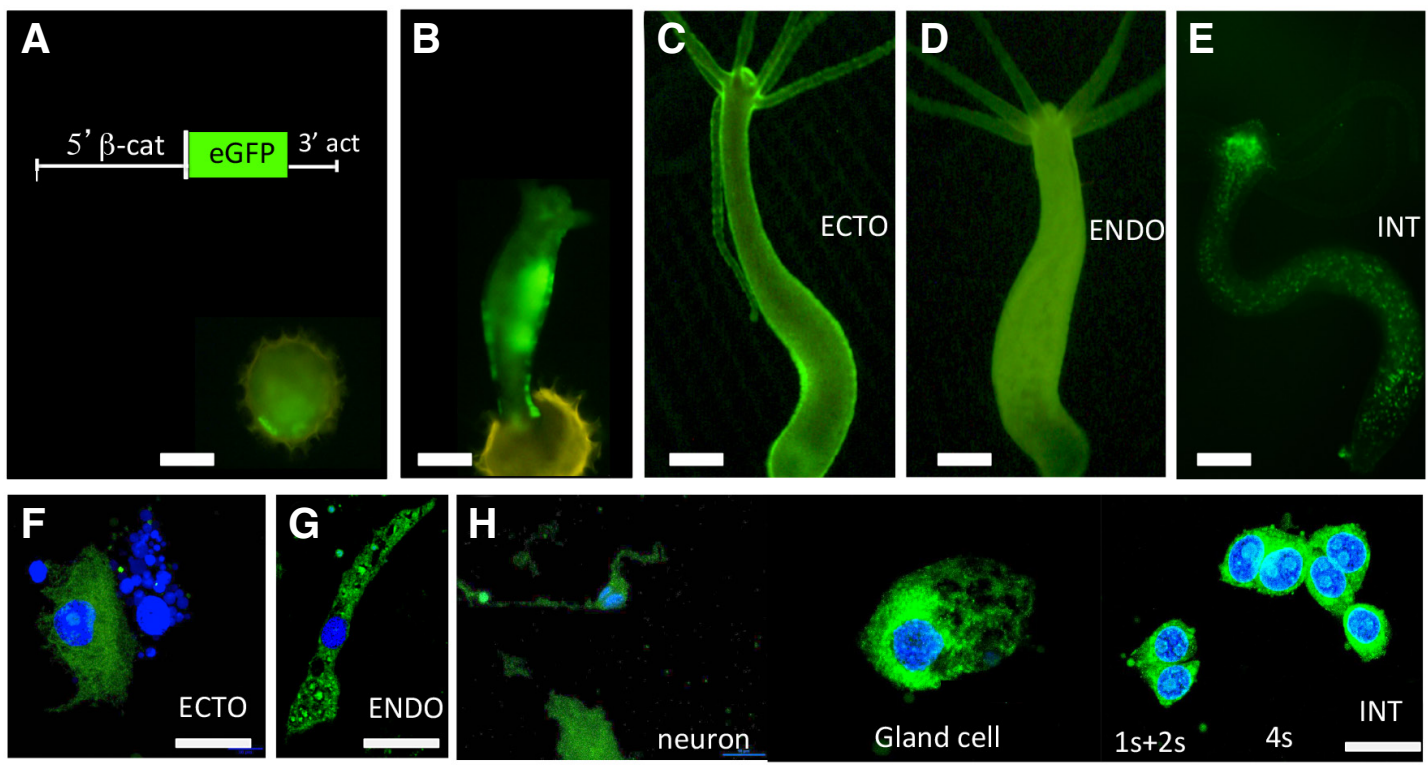

Fig. 1. $\boldsymbol{\beta}$-catenin promoter is active during development and in all adult cell lineage. (A) A Hydra embryo 2 weeks post injection of the recombinant plasmid shown in the upper part shows diffuse reporter expression, indicating activation of the $\beta$-cat promoter during embryo development. Reporter expression continues in the hatched polyp (B), and after clonal propagation founder polyps were selected in which eGFP was restricted in the ectoderm (C), in the endoderm (D), or in the interstitial cells (E). Homogeneous expression of $\beta$-cat driven eGFP in single cells obtained from ECTO (F), ENDO (G) and INT (H) lines was analysed by immunostaining on Hydra single cell suspensions, using anti-GFP antibody. Fluorescence images were acquired by laser scanning confocal microscopy. Scale bars, $500 \mu \mathrm{m}, \mathrm{A}-\mathrm{E} ; 20 \mu \mathrm{m}$ in F-G; $10 \mu \mathrm{m}$ in $\mathrm{H}$. 
of temporal resolution urges development of new tools, intended to in vivo monitoring the dynamic regulation of gene expression. Transgenic polyps expressing a $\beta$-cat fused to GFP under the actin promoter were developed in this direction (Gee et al., 2010). Although constitutive and ubiquitous expression of $\beta$-cat under a strong promoter contributed to deciphering $\beta$-cat role in the continuous maintenance and activity of the head organizer, it does not provide realistic information on $\beta$-cat physiological expression. For this reason here we produced a transgenic reporter Hydra expressing the eGFP reporter under the native $\beta$-cat promoter, containing all cis-elements sequences to drive expression in different physiological contexts, and exploited it to gain knowledge on $\beta$-cat controlled dynamics in living Hydra polyps. In order to characterize whole dynamic expression in vivo, we used Light Sheet Fluorescence Microscopy (LSFM), a technique that is particularly suitable for the reconstruction of an entire biological sample in 3D with single cell resolution (Santi, 2011). Taking advantage of the high acquisition speed and reduced photo-toxicity of LSFM we could record developmental and regeneration processes in Hydra during long-term time-lapse experiments. By monitoring adult homeostasis, budding, regeneration and forced activation of Wnt/ $\beta$-cat pathway by chemical compounds, we add new clues on known processes triggered by Wnt/ $\beta$-catenin signalling, and open the path to detect $\beta$-catenin modulation in many other physiological contexts.

\section{Results}

\section{Generation of Wnt/ $\beta$-cat reporter Hydra}

To generate $W n t / \beta$-cat reporter Hydra, a DNA fragment of $1143 \mathrm{bp}$ upstream of the transcriptional start of the $\beta$-cat gene, including 21 nucleotides of $\beta$-cat coding sequence (Fig. 1A), was cloned into the pHyVec13 vector (kind gift from $\mathrm{R}$. Steele), upstream and in-frame with the eGFP coding sequence, replacing the actin promoter. Promoter scanning by bioinformatics tool predicts multiple putative transcription factor binding sites within this regulative region (Fig. S1), as recently described in detail (Gufler et al., 2018). Other elements of the construct, including the 3'-flanking region downstream of the GFP coding sequence, remained intact. Before stable transformation, the DNA construct was tested by transient expression using a modified biolistic method (details are in the Supporting Information). eGFP fluorescence (Fig. S2) was detectable $96 \mathrm{~h}$

Fig. 2. $\beta$-cat driven eGFP expression in vivo recapitulates $\beta$-cat expression analyzed by in situ hybridization. In situ hybridization (A) showing $\beta$ cat expression during budding (Images with permission of (Hobmayer et al., 2000)). In the final stage of budding expression is detected in the apical hypostome. In vivo fluorescence imaging (B) of $\beta$ cat eGFP-ECTO transgenic polyp-showing $\beta$-cat reporter upregulation in the prospective budding zone, and then in the newly formed bud. A circular fluorescent pattern depicts the tentacle emerging domains. Upregulation persists in the new bud, throughoutbudding process. (C) LSFM Maximum Intensity Projections of stacks acquired during a time-lapse of the budding event. False colours (blue-green) are used to highlight the fluorescence increase in the budding region. post transfection, restricted to the hypostome, providing functional evidences of the engineered reporter construct in Hydra and confirming high Wnt/ $\beta$-cat signalling activity in this region.

Genetic transformation of Hydra vulgaris strain AEP embryos by microinjection was performed as previously described (Wittlieb et al., 2006). All embryos transfected showed a diffuse fluorescence, reflecting previously detected endogenous $\beta$-cat expression throughout embryogenesis (Frobius et al., 2003)(Fig. $1 \mathrm{~A})$. Out of 31 injected embryos 11 hatched, were initially mosaic, with patches of eGFP signal observed along the body column (Fig. 1B, Fig. S3, S4). Further, these founder polyps were clonally propagated and selected for enrichment of transgenic cells to form clonal fully-transgenic Hydra lines. All polyps demonstrated normal morphology and behaviour, indicating no adverse effects of embryo manipulation or eGFP expression. For further analysis three lines derived from a single founder (\#C3) were selected (Fig. 1C-E), expressing eGFP in ectoderm (ECTO), endoderm (ENDO) or interstitial lineage (INT). PCR analysis performed on polyps not expressing eGFP, derived from mosaic founders, showed the absence of exogenous eGFP DNA sequence (data not shown), indicating that the absence of fluorescence was due to the absence of the transgene and not to a position-dependent suppression of the transgene expression.

In fully transgenic ECTO and ENDO polyps reporter gene expression was detected throughout the polyp (Fig. 1C, D), recapitulating the endogenous expression of Hydra $\beta$-cat (Gee et al., 2010, Hobmayer et al., 2000). Single-cell suspensions obtained by maceration and immunostained with anti-GFP antibody showed homogeneous composition of ECTO transgenic polyps by only eGFP+ ectodermal epitheliomuscular cells, and ENDO polyps by only eGFP+ endodermal cells (Fig. 1F-G). In animals expressing $\beta$-cat-eGFP as a weak punctuated pattern (by in vivo whole imag-
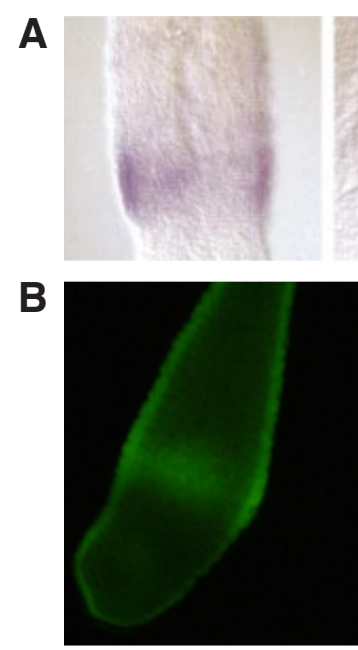

C

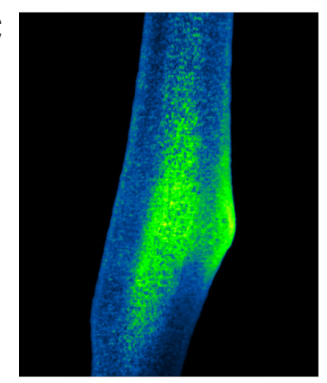

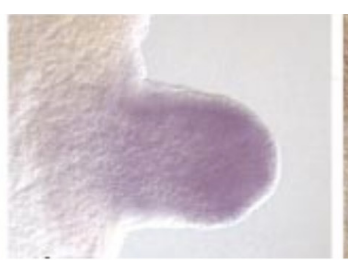
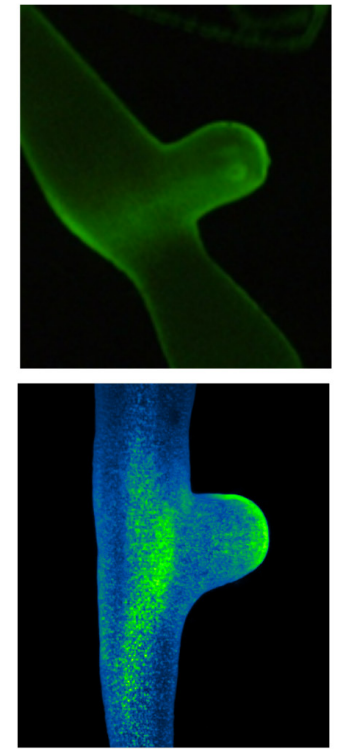
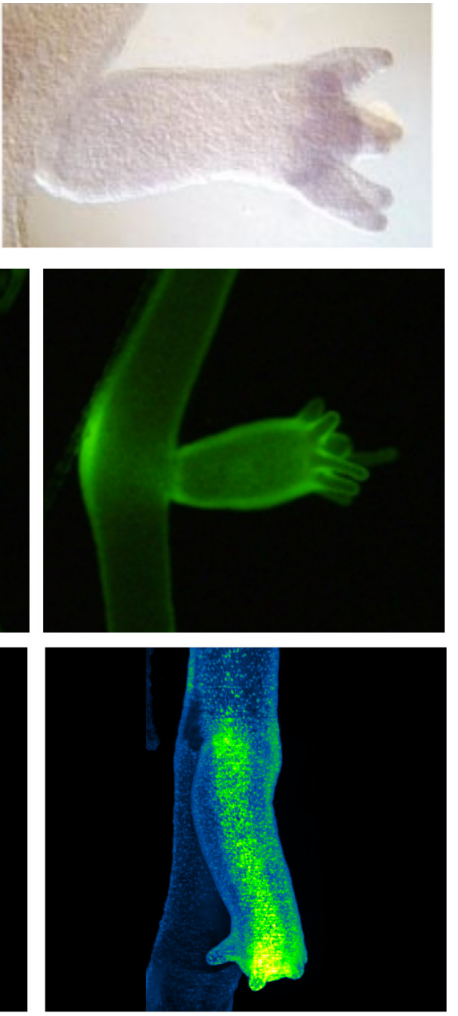

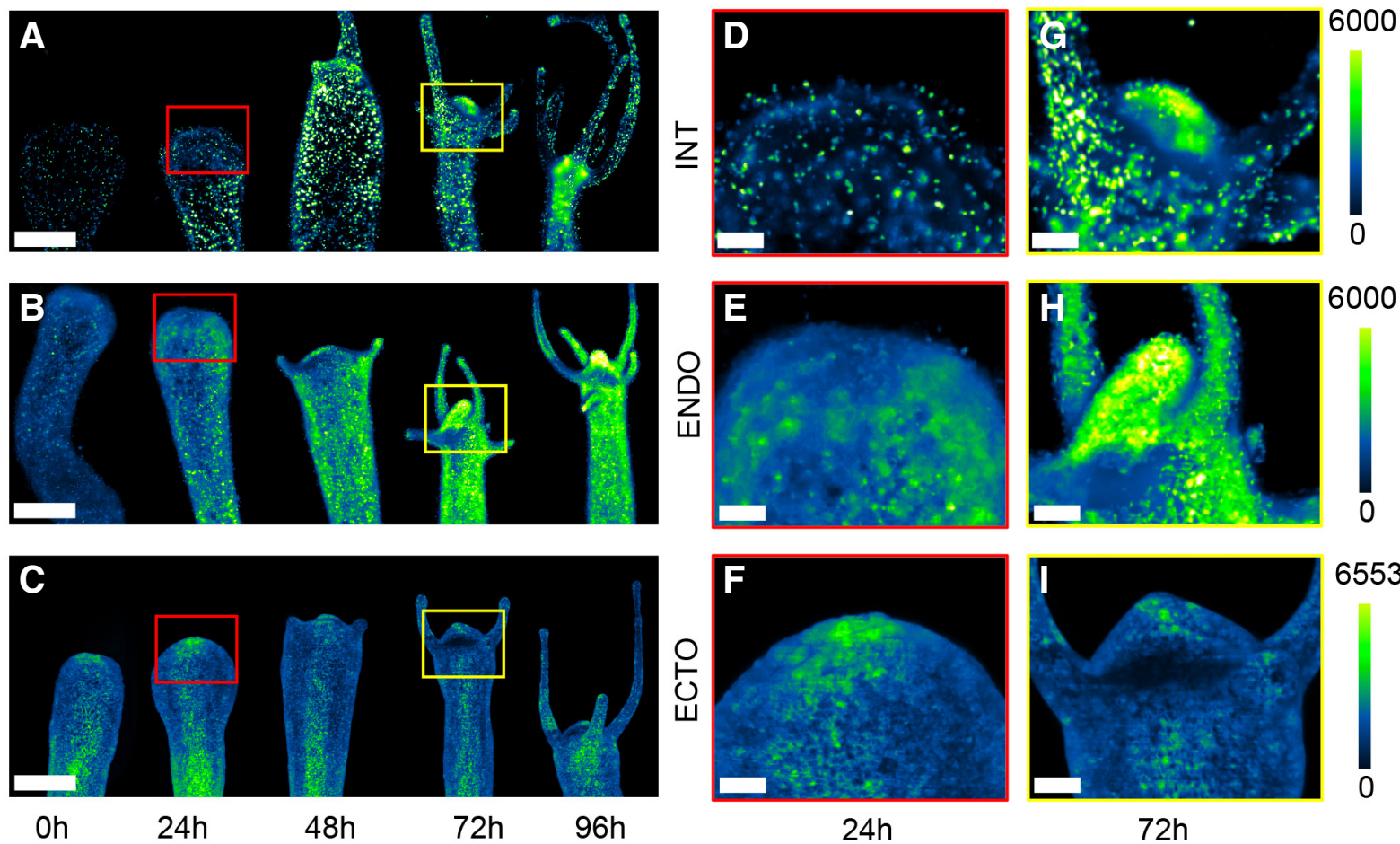

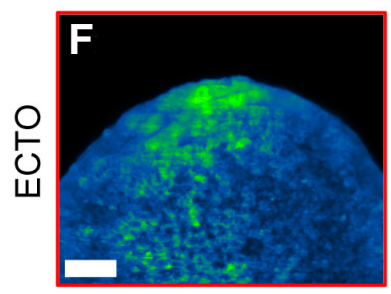

$24 \mathrm{~h}$

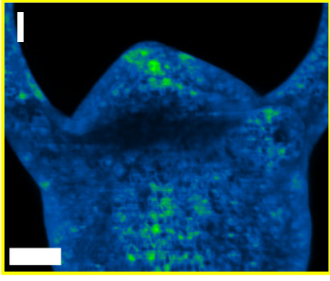

65535

$72 \mathrm{~h}$

Fig. 3. Time lapse analysis of $\boldsymbol{\beta}$-cat driven eGFP expression during regeneration. Activity of $\beta$-cat promoter was imaged in each cell lineage by acquiring $\beta$-cat-eGFP INT (A), ENDO (B) and ECTO (C) polyp during the whole process of regeneration. Details of the hypostomal region at $24 \mathrm{~h}$ and $72 \mathrm{~h}$ for INT polyp (D,G), ENDO (E,H), ECTO (F,I). Acquisitions are shown every $24 \mathrm{~h}$ from $0 \mathrm{~h}$ (right after amputation) to $96 \mathrm{~h}$ post amputation. Images are Maximum Intensity Projections of the acquired light sheet microscopy stacks. The images are shown in false colors. The color bar shows the fluorescence intensity in each pixel, indicating that the fluorescence signal emitted by ECTO is an order of magnitude higher than the one emitted by INT and ENDO. Scale bars are $500 \mu \mathrm{m}$ in $A, B, C$ and $100 \mu \mathrm{m}$ in $D, E, F, G, H, I$.

ing) (Fig. 1E), immunostaining revealed eGFP expression in all interstitial cell types, i.e. stem cells (1s, 2s) and their proliferating and differentiating derivatives (nematoblast nests, neurons, gland cells (Fig. 1H). In these polyps, referred to as INT, the fluorescence was enhanced in the endodermal hypostomal region (Fig. S5), probably due to the high concentration of gland cells in this region. Supplementary Fig. S6, S7 and S8 show whole animals and single sagittal sections of the acquired stacks of images relative to ECTO, ENDO and INT reporter lines, which provide unequivocal eGFP expression pattern per each cell lineage.

The reporter gene expression pattern, monitored in each line from establishment of transgenesis to date, did not change over time, suggesting the transgene stability and the possibility to study dynamic processes over long periods.

In sum, the created transgenic lines can be used further to monitor in vivo the dynamic transcriptional activity of $\beta$-cat promoter in different developmental processes independently in three cell lineages.

\section{Wnt/ $\beta$-cat signalling during budding}

Asexual reproduction in Hydra starts with the appearance of an evagination of the body column wall (ca 2/3-rds down the body column length), followed by elongation into a protrusion. After formation of a head with tentacles and foot at the apical and basal ends of the protrusion, the bud detaches. Both canonical and non canonical Wnt signalling participate in the tissue evagination process (Philipp et al., 2009) and $\beta$-cat reporter analysis may reveal important spatiotemporal clues on $\beta$-cat driven processes. To this aim, adult transgenic Hydras were monitored by fluorescence microscopy throughout the entire budding process. In the ECTO line, enhanced fluorescence expression was detected in a ring-like domain in the forthcoming budding zone, approximately 18-24 $\mathrm{h}$ before a tissue evagination becomes evident (Fig. 2B, Fig. S9). The enhanced fluorescence persisted in the emerging bud all along the budding process, and it was maintained until bud detachment, mirroring $\beta$-cat expression pattern detected by in situ hybridization (Fig. 2A) (Hobmayer et al., 2000). However, reporter gene expression was not limited to the apical region of the bud, but was clearly augmented throughout the body, suggesting extended promoter activity compared to that detected by in situ hybridization. A time-lapse acquisition by LSFM was then performed, capturing the whole expression dynamic of the fluorescence signal (Fig. 2C, Fig. S10). The enhancement of the eGFP expression in the gastric region persisted throughout the evagination process, being not limited to the budding region, but extending in the upper and lower regions, and throughout the whole bud length.

In the ENDO line a similar increase in the fluorescence in the budding region was observed, although less pronounced compared to the ECTO line, while in the interstitial line no change in the reporter gene expression was detectable, interstitial cells being thoroughly dispersed uniformly in the growing bud (data not shown). This evidence supports a major role played by $\beta$-cat expressing epithelial cells during morphogenesis. 


\section{$\beta$-cat promoter activity during regeneration}

Hydra polyps display a unique developmental plasticity among adult multicellular organisms: after amputation, they regenerate a missing part in few days (3-4 days in optimal culture conditions). The cellular and molecular events displayed by a head-regenerating tip have been exhaustively investigated (Bode, 2003, Bosch, 2007, Buzgariu et al., 2018, Chera et al., 2009, Galliot et al., 2006, Petersen et al., 2015). Here we analysed the full expression dynamic of the $\beta$-cat promoter in vivo, in regenerating heads of all transgenic lines by LSFM in time lapse experiments. Fig. 3 depicts the $\beta$-cat dynamics during regeneration in INT, ENDO and ECTO Hydra transgenic lines, from the time of amputation up to $96 \mathrm{~h}$ post amputation (p.a). Data were acquired every $40 \mathrm{~min}$ and are shown every $24 \mathrm{~h}$. The INT line (expressing reporter gene in interstitial cells and their derivatives) is characterized by single fluorescent cells evenly distributed throughout the body column and numerous fluorescent nerve cells, especially at the foot and peduncle regions (Fig. 3A, Fig. S5). Early stages p.a. show evident changes in the eGFP expressing cells, that are detectable both in intensity and spatial pattern, and are more pronounced in the gastric region (Fig. $3 A$ ). At later stages ( $48 \mathrm{~h}$ p.a.) the number of eGFP+ cells greatly increases throughout the whole body column up to the newly patterned hypostome and emerging tentacle buds. The massive contribution of $\beta$-cat expressing endodermally-located gland cells in the head regeneration appears evident from $72 \mathrm{~h} \mathrm{p}$.a. onwards. Fig. $3 \mathrm{~A}$ shows enhanced fluorescence in the hypostome at $96 \mathrm{~h}$, progressively decreasing toward the gastric region (Fig. S11). The active involvement of $\beta$-cat expressing endodermal cells during regeneration was revealed by the analysis of the ENDO line. The

A $\mathrm{Oh}$

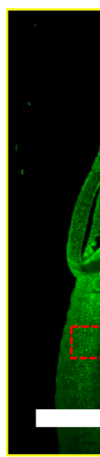

B

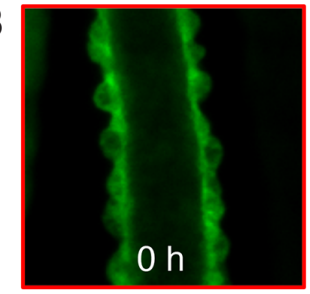

C

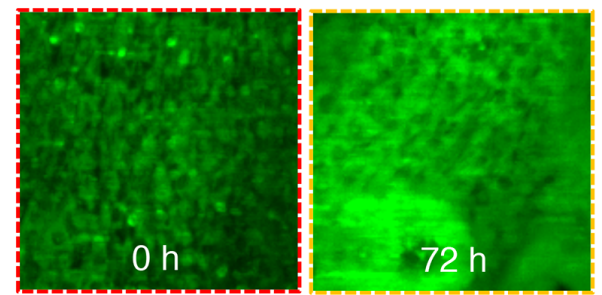

$24 \mathrm{~h}$
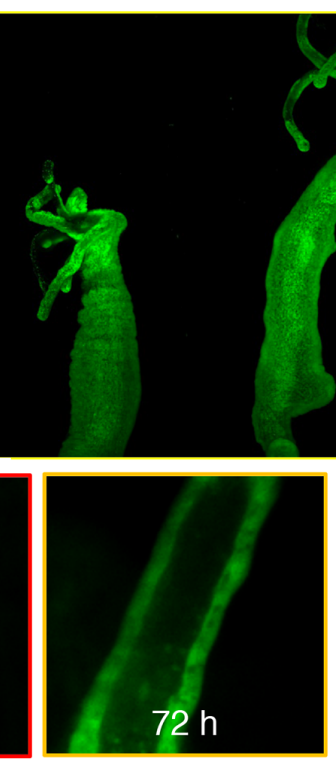

$48 \mathrm{~h}$

$60 \mathrm{~h}$

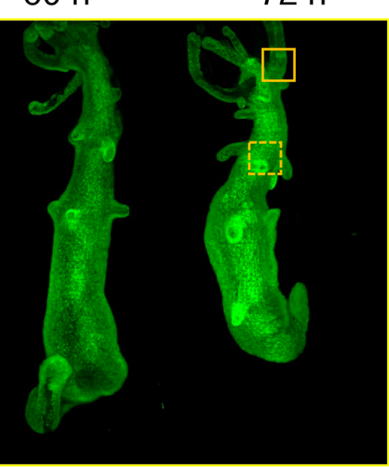

D

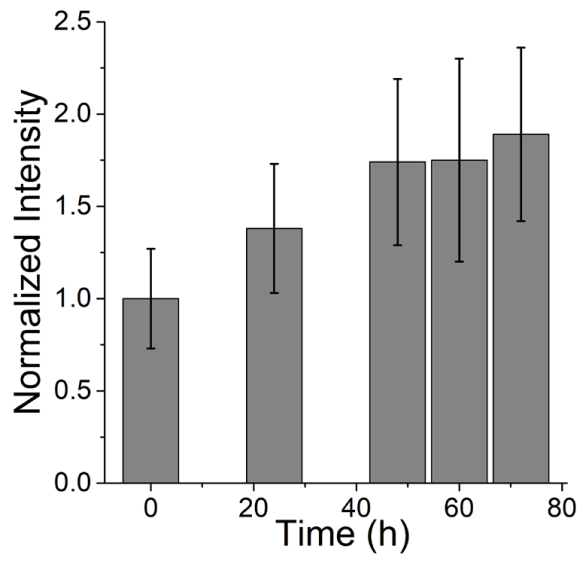

process of head regeneration of a ENDO polyp is presented in the Fig. 3B. A marked enhancement of an endodermal fluorescence was detectable since the beginning of the process. The signal intensity progressively increases all over the body, reaching the highest level in the hypostomal region, $96 \mathrm{~h}$ post amputation.

From analysis of the ECTO line during head regeneration (Fig. 3C), a moderate increase in eGFP+ expressing cells was detected after amputation in the hypostome and at higher extent at the gastric region level, which has not been reported by in situ hybridization. Optical sections of each transgenic line performed at the beginning and at the end of time lapse experiments (Fig. S12) show reporter gene expression being restricted to lineage specific cells. Altogether the time-lapse analysis of $\beta$-cat-eGFP expressing polyps, transgenic in single cell lineage, highlights the key role of endodermal cells in driving the regenerative process. This is in line with recent identification of $\beta$-cat target genes, all expressed in the endoderm and strongly upregulated during the early phase of head and foot regeneration (Gufer, 2018).

eGFP expression is upregulated in response to GSK-3 $\beta$ inhibition and correlates with $\beta$-cat expression in ectopic tentacles

To determine whether the $\beta$-cat-eGFP reporter responds to signals that trigger the Wnt pathway, we activated the Wnt pathway by inhibition of the glycogen synthase kinase- $3 \beta$ GSK3 using ALP (Leost et al., 2000). $\beta$-cat reporter animals were treated with ALP for $24 \mathrm{~h}$, and imaged at $24 \mathrm{~h}, 48 \mathrm{~h}$ and $72 \mathrm{~h}$ post treatment. In Fig. 4 images from a $\beta$-cat-eGFP ECTO polyp clearly show progressive increase of fluorescence throughout the body length and in the tissue organizing the new tentacles. Localised reporter expression before tentacle protrusion and throughout morphogenesis confirms the role of $\beta$-cat in molecular pre-patterning and differentiation of head specific structures. Sagittal section of the acquired stack for the ECTO polyp (Fig. 4 B,C) confirms that the activity of the $\beta$-cat promoter is confined to the ectodermal layer. Similarly, in ENDO polyps, eGFP fluorescence remains restricted to endodermal cells, indicating the lineage specific contribute to ectopic tentacle formation (Fig. S13). Time lapse LSFM performed on $\beta$-cat-eGFPINT polyps shows a slight increase of reporter expression both in the hypostome and in the body (Fig. S14).

Fig. 4. Temporal dynamics of $\beta$-catenin expression during Wnt signalling activation. $\beta$-cat-eGFP ECTO transgenic polyps were exposed $24 h$ to $5 \mu \mathrm{M} A L P$ and acquired with LSFM. Images are Maximum Intensity Projections of the acquired light sheet microscopy stacks, at $0 \mathrm{~h}, 24 \mathrm{~h}, 48 \mathrm{~h}, 60 \mathrm{~h}$ and $72 \mathrm{~h}$ from treatment(A). Details of the tentacle and body regions at the beginning (red squares) and at the end (yellow squares) of the acquisition are shown in (B) and (C), respectively. Fluorescence intensity as a function of time, quantified on the body region of ECTO polyps $(n=3)$ after segmentation of the LSFM data (D) 


\section{Discussion}

We generated fluorescent transgenic $\beta$-cat reporter strains of Hydra and performed an in depth characterization of its expression in adults during budding, regeneration and pharmacological activation of the Wnt pathway, dissecting the dynamic regulation of $\beta$-cat promoter activity in each cell lineage during these events. The reporter construct, comprising a fragment of the $\beta$-cat promoter driving eGFP expression, provides spatiotemporal information on endogenous $\beta$-cat promoter activity, substantially recapitulating the expression patterns obtained from in situ hybridization. eGFP reporter expression was detected in expected sites of $\beta$-cat promoter activity, i.e. apical tip of emerging buds and regenerating heads and in all cells of lineage-restricted transgenic line (ectodermal, endodermal and interstitial cells, throughout the body) which may account for the second function of $\beta$-cat in cellcell adhesion, described in Hydra and other cnidarians (Broun et al., 2005, Hobmayer et al., 2000, Wikramanayake et al., 2003). Additionally, novel sites of $\beta$-cat promoter activity were identified depicting the dynamic expression pattern in different physiological contexts. During development of a new bud, $\beta$-cat promoter was found active along the whole bud length, gradually extending from the emerging zone up to the bud apical end. During head regeneration the possibility to analyse the three separate lineage expressing lines allowed us to identify the endoderm as the tissue where the $\beta$-cat promoter is most active, and culminating at 96 $\mathrm{h}$. These findings agree with those previously obtained in early regenerates by in situ hybridization (Gee et al., 2010, Gufler et al., 2018, Hobmayer et al., 2000) showing $\beta$-cat up-regulation in the apical hypostome. However, marked differences were found at later stages, when in situ hybridization failed to detect $\beta$-cat expression, while in the present work $\beta$-cat promoter activity is observed up to $96 \mathrm{~h}$, when the differentiation of head structure takes place. The crucial role of endoderm in pre-patterning, but not later phases of regeneration is supported by recent identification of a set of endoderm specific genes (zebra, matrixin, cathepsinL1like, hv100202099, bmp-binding like) controlled by $\beta$-cat that may orchestrate the wound healing immediately post-dissection (Gufler et al., 2018). $\beta$-cat has been suggested to act also at translational level to dictate specific differentiation programs, although the underlying mechanisms have been not ruled out. Here we show that $\beta$-cat promoter activation is not transient, but controls and sustains also late differentiation programs.

The expression pattern of $\beta$-cat gene and the combined involvement of $\beta$-cat/TCF in the transcriptional activation of Wnt3 in Hydra were recently investigated by producing transgenic animals for the eGFP-tagged $\beta$-cat gene under the control of the $\beta$-cat promoter (Nakamura et al., 2011). In these animals $\beta$-cateGFP fusion protein was found expressed throughout the body, with nuclear localization in the hypostome. Upon ALP treatment, stabilization of $\beta$-cat-eGFP led to increased nuclear accumulation throughout the animal. In the transgenic animals generated in this work, forced activation of Wnt signalling led to the augmented expression of $\beta$-cat driven eGFP during pre-patterning and differentiation of ectopic tentacles, confirming the $\beta$-catenin central role in Wnt signalling.

It is worth emphasizing that the achievement of additional information was due not only to the possibility offered by transgenic Hydra to analyse gene expression in single tissue layers, but also to the imaging technique adopted. Indeed, using LSFM microscopy, we were able to capture, by continuously imaging the whole samples along time, the dynamic of Wnt signalling activation throughout physiological processes, accurately identifying all major sites of $\beta$-cat activity.

Aware of possible misleading interpretations of the observed expression pattern over long periods, due to high stability of eGFP protein, the reporter strains here produced revealed in real time the dynamic expression of $\beta$-cat gene in developmental and pharmacological contexts, opening the path to functional studies where $\beta$-cat involvement or modulation has not been postulated. Positive and negative regulators acting upstream of Wnt are being identified in vertebrates, and most common functional tests are performed by using luciferase/GFP reporter of $\beta$-cat-mediated transcriptional activation, i.e. TOPFLASH assays (Veeman et al., 2003), where reporters are driven by multiple copies of TCF binding sites. With the final aim to produce a similar reporter in Hydra, we used endogenous $\beta$-cat regulatory sequence to drive GFP expression, supported by prediction of regulatory cis-elements within this region (Gufler et al., 2018), including the TCF binding site GTTTGAT, i.e. TCF-site 3 core previously identified (Nakamura et al., 2011). Considering the central role played by $\mathrm{Wnt} / \beta$-cat signalling pathway, including cellular proliferation, differentiation, migration, polarity, and regeneration (van Amerongen and Nusse, 2009), $\beta$-cat-GFP reporter lines may be used as ideal genetic background to investigate cross-talk between different signalling pathways, to identify upstream regulators, such as Crypto, Sp5 (Huggins et al., 2017, Morkel et al., 2003) or responsiveness of $\beta$-cat transcription to any external chemical or physical stimulation including temperature, feeding regime, presence of microbes. These studies may shed light on how developmental programs are influenced by environment (Bosch et al., 2014), and in which condition these programs are reactivated. This work demonstrated the enhancement of $\beta$-cat promoter activity during budding, regeneration and ALP treatment as first functional evaluations. An ongoing study using these lines is aimed to evaluate the action range of Wnt $/ \beta$-cat signalling. Indeed, when using ALP for forced activation of Wnt signalling all cells are equally exposed to the drug and ectopic tentacles emerge along the whole body length. What would be an outcome of local delivery of ALP delivery in certain cells? How neighbour cells would respond to $\mathrm{Wnt} / \beta$-cat activation and how this effect would be context dependent? In a previous work we produced light responsive microcapsules loaded with ALP and showed their functionality to induce ectopic tentacle formation all over the body (Ambrosone et al., 2016). The reporter lines here produced will provide important clues on systemic effect of Wnt signalling activation in single cells, and on the kinetic of this process, from the time of ALP delivery to GFP appearance and tentacle patterning. Finally, the construct that we engineered may be used as valuable backbone for further genetic manipulations in the context of Wnt/ß-cat -activity.

\section{Materials and Methods}

Hydra vulgaris strain AEP polyps were asexually cultured in Hydra medium ( $\left.1 \mathrm{mM} \mathrm{CaCl}_{2}, 0.1 \mathrm{mM} \mathrm{NaHCO}_{3}, \mathrm{pH}^{7}\right)$ at $18^{\circ} \mathrm{C}$ and fed three times per week with freshly hatched Artemia salina nauplii. For regeneration experiments, $24 \mathrm{~h}$ starved polyps were bisected in the upper gastric region and continuously monitored by fluorescence microscopy (see below). Forced activation of Wnt signalling was obtained by treating polyps with 
$5 \mu \mathrm{M}$ ALP solution for 24 hours, as previously described (Broun et al., 2005). Thereafter, polyps were rinsed several times and monitored by fluorescence microscopy.

\section{Generation of transgenic Hydra}

To generate the $\beta$-cat-GFP construct, 1143 bp of $\beta$-cat promoter (GenBank no. U38624.1) was amplified from genomic DNA using Platinum High Fidelity Taq polymerase (Invitrogen) and cloned into the Bglll/Pstl sites of $p H y V e c 13$ vector (kindly gifted by R.Steel, Irvine), replacing the actin promoter. Primers used for the PCR reaction were: FORW $\beta$-CAT: 5'-GGAAGATCTTCTTAGTTATCCGTATATCG-3'; REV $\beta$-CAT: 5'-ATGCTGCAGTACCAGTTGAATCCTCCAT-3'. The recombinant construct was sequenced to confirm its structure.

Transient expression of $\beta$-cat-eGFP plasmid DNA in Hydra vulgaris was performed by biolistic method, as previously described with minor modifications (Scotti and Cardi, 2012). Gold microcarriers (0.6 $\mu \mathrm{m}$, BioRad) were coated with plasmid DNA and spread over the center of macrocarrier. DNA delivery was achieved using a PDS-1000/He TM Gene Gun delivery system (Bio-Rad, Hercules, CA, USA). The Petri dish containing the Hydra was placed into the target plate holder and then in the third slot $(6 \mathrm{~cm})$ from the bottom of PDS chamber. A 1100 psi helium and 27-28 in. of Hg vacuum pressure were used for shooting. After shooting, Hydra were maintained in culture and continuously monitored for eGFP expression.

Stable genetic transformation of Hydra vulgaris strain AEP embryos was achieved by microinjection as previously described (Wittlieb et al., 2006). Animals were genotyped by PCR. Out of 31 injected embryos 11 hatched, and 7 transgenic founder lines ( $\beta$-cat-eGFP C3, C2, C6, A1, A2, B3, B2) were established exhibiting similar patterns in each of the cell lineages (ectoderm, endoderm and interstitial cells) but different levels of reporter expression. All lines analysed in this work during budding, regeneration and pharmacological treatment were obtained by clonal propagation of the founder line $\mathrm{C} 3$.

\section{Fluorescence immunostaining}

To confirm the eGFP cell type composition of each transgenic line immunocytochemistry using anti-GFP antibody was performed on single cells. Fixed Hydra cells were first obtained by maceration of $\beta$-cat-GFP transgenic polyps as described (David, 1973). Then the macerates were spread on slides, washed and permeabilized with PBS-Tween $0,1 \%$ for 1 h. Cells were incubated with a 1:200 dilution of mouse anti-GFP antibody in BSA-T for $3 \mathrm{~h}$ at room temperature and after washing incubated with a 1:1000 dilution of Alexa Fluor 488 labelled Goat anti-mouse secondary antibody for $1 \mathrm{~h}$ at room temperature. Cells were washed twice in PBS and slides mounted using Vectashield antifade mounting medium with DAPI (Vector Laboratories, Inc. CA).

\section{Wide field imaging}

Dynamics of reporter gene expression were analysed by in vivo imaging. Initial observations were performed by fluorescence microscopy using an inverted microscope (Axiovert 100, Zeiss) equipped with a digital color camera (Olympus, DP70) and fluorescence filter sets (BP450-490/FT510/ LP515). The software system Cell F(Olympus) was used for imaging analysis. Laser-scanning confocal data were acquired by using Nikon Eclipse Ti2. Pictures were taken by using Nikon A1R digital cameras.

\section{Light sheet fluorescence microscopy}

In order to observe regeneration and budding a LSFM setup (Candeo et al., 2016) was adapted to specifically image Hydra in vivo. In LSFM the sample is illuminated with a thin plane of laser light and the fluorescence is collected perpendicularly to the excitation axis through a fast CMOS camera, providing optical sectioning. The sample is rapidly scanned through the illumination plane (light sheet), hence a 3D volume is recorded. In details, a single-mode fiber-coupled laser emitting at $478 \mathrm{~nm}$ (MBL-FN-473 $\mathrm{CNI}$ ), is collimated and then focused in order to form a light sheet on the sample through a cylindrical lens $(f=75 \mathrm{~mm})$. The optical power of c.a.
$6 \mathrm{~mW}$ is split into two beams counter-propagating across the sample (Candeo et al., 2016). The fluorescence emitted by the sample is filtered and collected with a $4 \mathrm{X}$ objective lens (Nikon, PlanFluor $\mathrm{NA}=0.13$ ) and a band pass filter, which in combination with a tube lens create an image at the detector (ORCA Flash 4.0, Hamamatsu). The lateral resolution of the system is approximately $2 \mu \mathrm{m}$ and the axial resolution (given by the waist of the focused light sheet) is approximately $15 \mu \mathrm{m}$. The sample is placed in a cuvette filled with water and kept in a $0.8 \mathrm{~mm}$ diameter Fluorinated Ethylene Propylene (FEP) similarly to the protocol described in (Bassi et al., 2015). Here Hydra are not anesthetized and in order to avoid artefacts induced by the movements of the sample, the acquisition time was set to $30 \mathrm{~ms} / \mathrm{plane}$, in order to collect an entire LSFM stack (c.a. 150 planes over a thickness of $0.8 \mathrm{~mm}$ ) in less than $5 \mathrm{~s}$. During time lapse experiments the measurements were repeated every c.a. $40 \mathrm{~min}$. In these conditions photo-bleaching was negligible.

\section{Fluorescence quantification}

Data acquired with LSFM are processed in Matlab and visualized with Fiji. Image stacks are automatically segmented, using a threshold (Fig. S15). Then the software computes the average fluorescence intensity on a 50X50X50um volume selected by the user: the sum of the intensity over the entire volume is calculated and divided by the total volume. The voxel outside the segmented volume are excluded from the calculation automatically.

\section{Acknowledgements}

Technical assistance of Mrs. L. Sannino (IBBR-CNR, Portici) with biolistic experiment is gratefully acknowledged. We also thank Giuseppe Marino and Ilaria Panico (ISASI-CNR, Pozzuoli) for technical assistance with animal culture. This work was partially supported by Laserlab- Europe (EU-H2020 654148).

\section{References}

AMBROSONE, A., MARCHESANO, V., CARREGAL-ROMERO, S., INTARTAGLIA, D. PARAK, W.J. and TORTIGLIONE, C. (2016). Control of Wnt/beta-Catenin Signalling Pathway in vivo via Light Responsive Capsules. ACS Nano 10: 4828-4834.

ANASTAS, J.N. and MOON, R.T. (2013). WNT signalling pathways as therapeutic targets in cancer. Nat Rev Cancer 13: 11-26.

BARKER, N. and CLEVERS, H. (2006). Mining the Wnt pathway for cancer therapeutics. Nature reviews. Drug discovery 5: 997-1014.

BASSI, A., SCHMID, B. and HUISKEN, J. (2015). Optical tomography complements light sheet microscopy for in toto imaging of zebrafish development. Development 142: 1016-1020.

BODE, H.R. (2003). Head regeneration in Hydra. Dev Dyn 226: 225-236.

$\mathrm{BOSCH}$, T.C. (2007). Why polyps regenerate and we don't: towards a cellular and molecular framework for Hydra regeneration. Dev Biol 303: 421-433.

BOSCH, T.C.G., ADAMSKA, M., AUGUSTIN, R., DOMAZET-LOSO, T., FORET, S., FRAUNE, S., FUNAYAMA, N., GRASIS, J., HAMADA, M., HATTA, M. et al., (2014). How do environmental factors influence life cycles and development? An experimental framework for early-diverging metazoans. Bioessays 36: 1185-1194.

BROUN, M., GEE, L., REINHARDT, B. and BODE, H.R. (2005). Formation of the head organizer in hydra involves the canonical Wnt pathway. Development 132: 2907-2916.

BUZGARIU, W., WENGER, Y., TCACIUC, N., CATUNDA-LEMOS, A.P. and GALLIOT B. (2018). Impact of cycling cells and cell cycle regulation on Hydra regeneration. Dev Biol 433: 240-253.

CANDEO, A., SANA, I., FERRARI, E., MAIURI, L., D'ANDREA, C., VALENTINI, G and BASSI, A. (2016). Virtual unfolding of light sheet fluorescence microscopy dataset for quantitative analysis of the mouse intestine. J Biomed Opt 21: 56001.

CHERA, S., GHILA, L., DOBRETZ, K., WENGER, Y., BAUER, C., BUZGARIU, W., MARTINOU, J.C. and GALLIOT, B. (2009). Apoptotic cells provide an unexpected source of Wnt3 signaling to drive hydra head regeneration. Dev Cell 17: 279-289.

CLEVERS, H. (2006). Wnt/beta-catenin signaling in development and disease. Cell 127: 469-480. 


\section{R. Iachetta et al.}

DAVID, C.N. (1973). A quantitative method for maceration of hydra tissue. Wilhelm Roux Arch Entwickl Mech Org 171: 259-268.

FROBIUS, A.C., GENIKHOVICH, G., KURN, U., ANTON-ERXLEBEN, F. and BOSCH, T.C. (2003). Expression of developmental genes during early embryogenesis of Hydra. Dev Genes Evol 213: 445-455.

GALLIOT, B., MILJKOVIC-LICINA, M., DE ROSA, R. and CHERA, S. (2006). Hydra, a niche for cell and developmental plasticity. Semin Cell Dev Biol 17: 492-502.

GEE, L., HARTIG, J., LAW, L., WITTLIEB, J., KHALTURIN, K., BOSCH, T.C. and BODE, H.R. (2010). beta-catenin plays a central role in setting up the head organizer in hydra. Dev Biol 340: 116-124.

GUDER, C., PHILIPP, I., LENGFELD, T., WATANABE, H., HOBMAYER, B. and HOLSTEIN, T.W. (2006). The Wnt code: cnidarians signal the way. Oncogene 25: $7450-7460$.

GUFLER, S., ARTES, B., BIELEN, H., KRAINER, I., EDER, M.K., FALSCHLUNGER, J., BOLLMANN, A., OSTERMANN, T., VALOVKA, T., HARTL, M. et al., (2018). beta-Catenin acts in a position-independent regeneration response in the simple eumetazoan Hydra. Dev Biol 433: 310-323.

HOBMAYER, B., RENTZSCH, F., KUHN, K., HAPPEL, C.M., VON LAUE, C.C., SNYDER, P., ROTHBACHER, U. and HOLSTEIN, T.W. (2000). WNT signalling molecules act in axis formation in the diploblastic metazoan Hydra. Nature 407: 186-189.

HOBMAYER, E., HATTA, M., FISCHER, R., FUJISAWA, T., HOLSTEIN, T.W. and SUGIYAMA, T. (1996). Identification of a Hydra homologue of the beta-catenin/ plakoglobin/armadillo gene family. Gene 172: 155-159.

HOLSTEIN, T.W. (2012). The evolution of the Wnt pathway. Cold Spring Harb Perspect Biol 4: a007922.

HUGGINS, I.J., BOS, T., GAYLORD, O., JESSEN, C., LONQUICH, B., PURANEN, A., RICHTER, J., ROSSDAM, C., BRAFMAN, D., GAASTERLAND, T. et al., (2017). The WNT target SP5 negatively regulates WNT transcriptional programs in human pluripotent stem cells. Nat Commun 8: 1034.

LENGFELD, T., WATANABE, H., SIMAKOV, O., LINDGENS, D., GEE, L., LAW, L., SCHMIDT, H.A., OZBEK, S., BODE, H. and HOLSTEIN, T.W. (2009). Multiple Wnts are involved in Hydra organizer formation and regeneration. Dev Bio/330: 186-199.

LEOST, M., SCHULTZ, C., LINK, A., WU, Y., BIERNAT, J., MANDELKOW, E., BIBB, J., SNYDER, G., GREENGARD, P., ZAHAREVITZ, D. et al., (2000). Paullones are potent inhibitors of glycogen synthase kinase-3beta and cyclin-dependent kinase 5/p25. Eur. J. Biochem. 267: 5983-5994.

LIM, X. and NUSSE, R. (2013). Wnt signaling in skin development, homeostasis, and disease. Cold Spring Harb Perspect Biol. 5: a008029.
MACDONALD, B., TAMAI, K. and HE, X. (2009). Wnt/beta-catenin signaling: components, mechanisms, and diseases. Dev. Cell 17: 9-26.

MCMAHON, A.P. and MOON, R.T. (1989). Ectopic expression of the proto-oncogene int-1 in Xenopus embryos leads to duplication of the embryonic axis. Cell 58 : 1075-1084.

MORKEL, M., HUELSKEN, J., WAKAMIYA, M., DING, J., VAN DE WETERING, M., CLEVERS, H., TAKETO, M.M., BEHRINGER, R.R., SHEN, M.M. and BIRCHMEIER, W. (2003). Beta-catenin regulates Cripto- and Wnt3-dependent gene expression programs in mouse axis and mesoderm formation. Development 130: 6283-6294

NAKAMURA, Y., TSIAIRIS, C.D., OZBEK, S. and HOLSTEIN, T.W. (2011). Autoregulatory and repressive inputs localize Hydra Wnt3 to the head organizer. Proc Nat Acad Sci USA 108: 9137-9142.

PETERSEN, H.O., HOGER, S.K., LOOSO, M., LENGFELD, T., KUHN, A., WARNKEN U., NISHIMIYA-FUJISAWA, C., SCHNOLZER, M., KRUGER, M., OZBEK, S. et al., (2015). A Comprehensive Transcriptomic and Proteomic Analysis of Hydra Head Regeneration. Mol Biol Evol 32: 1928-1947.

PHILIPP, I., AUFSCHNAITER, R., OZBEK, S., PONTASCH, S., JENEWEIN, M., WATANABE, H., RENTZSCH, F., HOLSTEIN, T.W. and HOBMAYER, B. (2009). $\mathrm{Wnt} /$ beta-catenin and noncanonical Wnt signaling interact in tissue evagination in the simple eumetazoan Hydra. Proc Natl Acad Sci USA 106: 4290-4295.

SANTI, P.A. (2011). Light sheet fluorescence microscopy: a review. J Histochem Cytochem 59: 129-138.

SCOTTI, N. and CARDI, T. (2012). Plastid Transformation as an Expression Tool for Plant-Derived Biopharmaceuticals. Transgenic Plants: Methods and Protocols, Second Edition 847: 451-466.

VAN AMERONGEN, R.E. and NUSSE, R. (2009). Towards an integrated view of Wnt signaling in development. Development 136: 3205-3214.

VEEMAN, M.T., SLUSARSKI, D.C., KAYKAS, A., LOUIE, S.H. and MOON, R.T (2003). Zebrafish prickle, a modulator of noncanonical Wnt/Fz signaling, regulates gastrulation movements. Curr Biol 13: 680-685

WIKRAMANAYAKE, A.H., HONG, M., LEE, P.N., PANG, K., BYRUM, C.A., BINCE, J.M., XU, R. and MARTINDALE, M.Q. (2003). An ancient role for nuclear beta-catenin in the evolution of axial polarity and germ layer segregation. Nature 426: 446-450.

WITTLIEB, J., KHALTURIN, K., LOHMANN, J.U., ANTON-ERXLEBEN, F. and BOSCH, T.C. (2006). Transgenic Hydra allow in vivo tracking of individual stem cells during morphogenesis. Proc Natl Acad Sci USA 103: 6208-6211.

WONG, G.T., GAVIN, B.J. and MCMAHON, A.P. (1994). Differential transformation of mammary epithelial cells by Wnt genes. Mol Cell Biol 14: 6278-6286. 


\section{Further Related Reading, published previously in the Int. J. Dev. Biol.}

Stemness in Hydra - a current perspective

Bert Hobmayer, Marcell Jenewein, Dominik Eder, Marie-Kristin Eder, Stella Glasauer, Sabine Gufler, Markus Hartl and Willi Salvenmoser Int. J. Dev. Biol. (2012) 56: 509-517

https://doi.org/10.1387/ijdb.113426bh

Interstitial stem cells in Hydra: multipotency and decision-making Charles N. David

Int. J. Dev. Biol. (2012) 56: 489-497

https://doi.org/10.1387/ijdb.113476cd

Hydra, a model system to trace the emergence of boundaries in developing eumetazoans Angelika Böttger and Monika Hassel

Int. J. Dev. Biol. (2012) 56: 583-591

https://doi.org/10.1387/ijdb.113454ab

Frontiers in fluorescence microscopy

José Rino, José Braga, Ricardo Henriques and Maria Carmo-Fonseca

Int. J. Dev. Biol. (2009) 53: 1569-1579

https://doi.org/10.1387/ijdb.072351jr

Wnt signaling in hydroid development: ectopic heads and giant buds induced by GSK-3beta inhibitors

Werner Müller, Uri Frank, Regina Teo, Ofer Mokady, Christina Guette and Günter Plickert Int. J. Dev. Biol. (2007) 51: 211-220

https://doi.org/10.1387/ijdb.062247wm

5 yr ISI Impact Factor $(2016)=2.421$
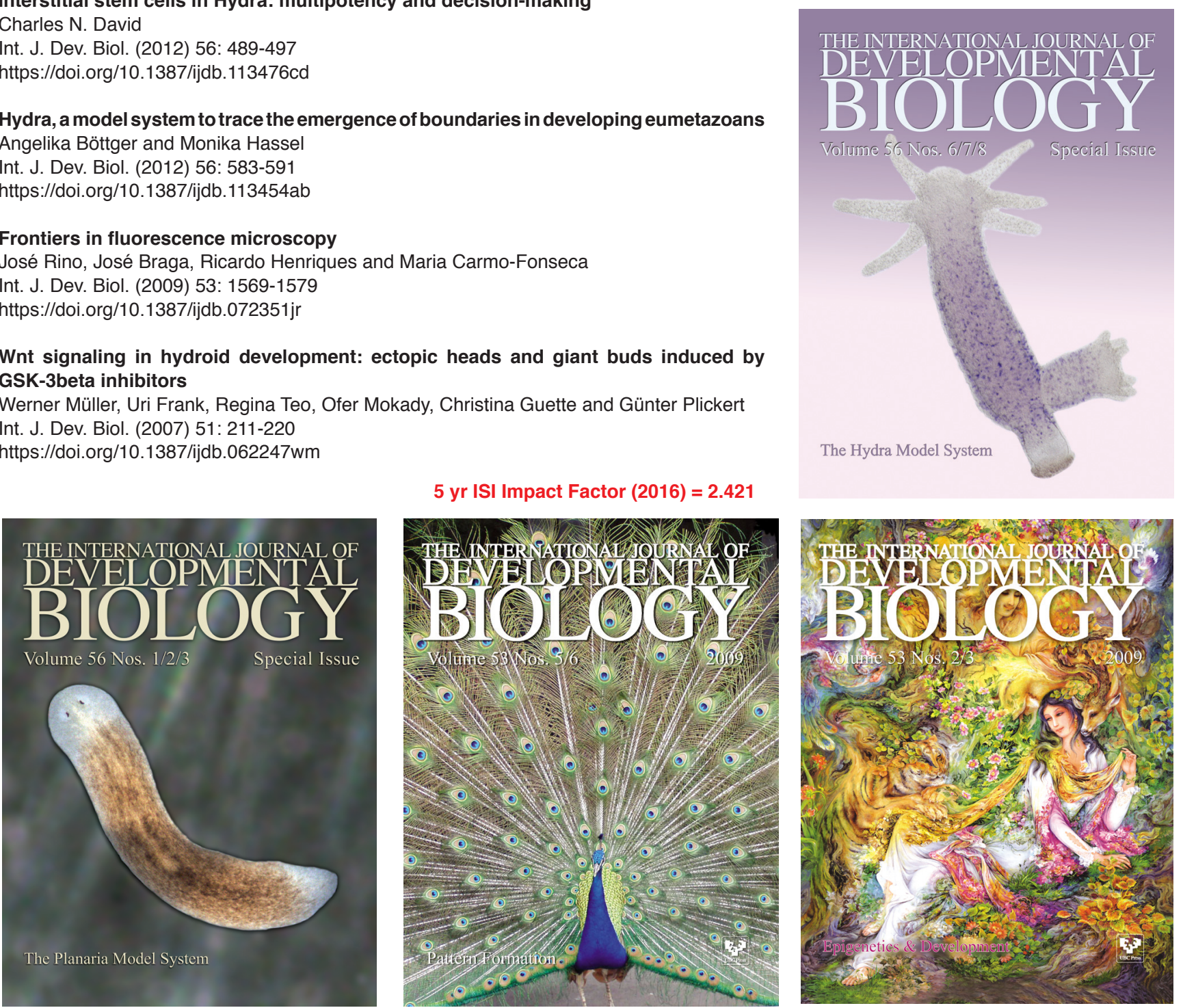